PROCEEDINGS OF THE AMERICAN MATHEMATICAL SOCIETY

Volume 127, Number 3, March 1999, Pages 861-874

S 0002-9939(99)04913-8

\title{
CHARACTERIZATION BASED ON CONDITIONAL EXPECTATIONS OF ADJACENT ORDER STATISTICS: A UNIFIED APPROACH
}

\author{
M. FRANCO AND J. M. RUIZ \\ (Communicated by Wei Y. Loh)
}

\begin{abstract}
In this paper, we show a unified approach to the problem of characterizing general distribution functions based on the conditional expectation between adjacent order statistics, $\xi(x)=E\left(h\left(X_{r, n}\right) \mid X_{r+1, n}=x\right)$ or $\bar{\xi}(x)=E\left(h\left(X_{r+1, n}\right) \mid X_{r, n}=x\right)$, where $h$ is a real, continuous and strictly monotonic function. We have the explicit expression of the distribution function $F$ from the above order mean function, $\xi$ and $\bar{\xi}$, and we give necessary and sufficient conditions so that any real function can be an order mean function. Our results generalize the results given for the discrete, absolutely continuous and continuous cases. Further, we show stability theorems for these characterizations.
\end{abstract}

\section{INTRODUCTION}

The problem of characterizing distributions through conditional expectations of adjacent order statistics has been of increasing interest during the last two decades due to its several applications, for example, the study of $(n-r)$-out-of- $n$ systems. A $(n-r)$-out-of- $n$ system consists of $n$ independent and identically distributed components, and it works as long as at least $(n-r)$ components are working. If $X_{i}$ represents the lifetime of the $i$ th component, $i=1,2, \ldots, n$, the survival function of the $(n-r)$-out-of- $n$ system is the same as that of the $(r+1)$ th order statistics $X_{r+1, n}$ from this sample of $n$ random variables. Hence, the results obtained for order statistics hold for $(n-r)$-out-of- $n$ systems, so the study of order statistics plays an important role in reliability theory. Recently, the order statistics have been extensively studied in the literature to characterize particular distributions and families of distributions, for instance Arnold [1], Arnold et al. [2], El-Neweihi and Govindarajulu [4], Franco and Ruiz [6, 7], Khan and Abu-Salih [9], Nagaraja $[11,12]$ and Ouyang $[13,14]$.

Let $X_{1}, X_{2}, \ldots, X_{n}$ be a set of $n$ independent and identically distributed random variables with increasing and right continuous distribution function $F$, and support $(\alpha, \beta)$, i.e., $\lim _{x \rightarrow \alpha+} F(x)=0, \lim _{x \rightarrow \beta-} F(x)=1$ and $0<F(x)<1$ for all $x \in(\alpha, \beta)$. And let $X_{1, n}, X_{2, n}, \ldots, X_{n, n}$ be the associated order statistics with $X_{r, n}<X_{r+1, n}$, which prevents the case of ties when $F$ is discrete, so the event $\left\{X_{r, n}=X_{r+1, n}\right\}$

Received by the editors June 5, 1997.

1991 Mathematics Subject Classification. Primary 62E10, 62G30, 60 E05.

Key words and phrases. Order statistics, $(n-r)$-out-of- $n$ system.

This work was partially supported by DGES (MEC), Grant PB96-1105. 
has zero probability when $F$ is discrete, and the $X_{r, n}$ 's possess Markovian structure (see Arnold et al. [3] and Nagaraja [10]).

For each $x \in D=\{x \in \mathbb{R}: F(x-)>0\}$, and $h$ is a given real, continuous and strictly monotonic function, we define the order mean function by

$$
\xi(x)=E\left(h\left(X_{r, n}\right) \mid X_{r+1, n}=x\right)
$$

or analogously, by

$$
\bar{\xi}(x)=E\left(h\left(X_{r+1, n}\right) \mid X_{r, n}=x\right)
$$

for each $x \in \bar{D}=\{x \in \mathbb{R}: F(x)<1\}$, where $1 \leq r<n$.

Hence, we propose two problems: (a) To obtain the parent distribution, given the order mean function. (b) To characterize the set of order mean functions.

These problems have been solved for particular expressions of $\xi$ and $\bar{\xi}$, when the distribution function $F$ is discrete, absolutely continuous or continuous.

For instance, in the discrete case: using $\bar{\xi}(x)=a x+b$ when $h(x)=x$, Nagaraja [12] characterizes Waring, geometric and generalized hypergeometric type distributions, and Franco and Ruiz [7] solve the above problems. In the absolutely continuous case: Rogers [15] obtains the exponential distribution when $h(x)=x$ and $\bar{\xi}(x)=x+b$; Ferguson [5] characterizes the parent distribution when $h(x)=x$ and $\xi$ or $\bar{\xi}$ is linear; Nagaraja [11] proves that, to $h(x)=x$, if both $\xi$ and $\bar{\xi}$ are linear, then $F$ is a uniform type distribution; and Khan and Abu-Salih [9] get some distributions by $\xi(x)=a h(x)+b$ or $\bar{\xi}(x)=c h(x)+d$, and they show one inversion formula when $h$ and $F$ are derivable. In the continuous case: Ouyang [13] characterizes the uniform distribution when $h(x)=x$ and $\bar{\xi}(x)=a x+b$ where $a=(n-r) /(n-r+1)$; Ouyang [14] obtains the distribution function by $\xi(x)=h(x)+c$ or $\bar{\xi}(x)=h(x)+c$ when $h$ is derivable and its derivative is continuous, and Franco and Ruiz [7] solve the above problems.

Since in characterization, emphasis is placed on obtaining results under the weakest assumptions on the distributions being characterized, we find it useful to prove stronger versions of these known results; for example, a characterization that requires a distribution be discrete or continuous is not as appealing as one that requires it only be a distribution function.

The main objective of the present paper is to solve the former problems in the case of arbitrary probability distributions. We give a unified approach for discrete, absolutely continuous and continuous cases, which will allow us to characterize mixture probability distributions of discrete and continuous distributions. Likewise, the characterization results given in the discrete, absolutely continuous and continuous cases, are particular cases of this work.

In Section 2, we consider corresponding questions to these objectives, and we give some technical results of the Riemann-Stieltjes product integral (RSPI) which will be useful in the following sections. In Section 3, we show general properties of the order mean functions. In Section 4, we obtain inversion formulas, i.e., explicit expressions of the distribution based on the order mean functions. In Section 5, we characterize the family of the order mean functions given necessary and sufficient conditions such that any real function is an order mean function. In Section 6, we obtain some stability theorems for these characterizations. Finally, in Section 7, we give some examples of characterization of mixture distributions. 


\section{TeChnical Results}

Let $\mathcal{F}$ be the set of the distribution functions $F$ satisfying $\int_{(\alpha, x)} h(y) d(F(y))^{r}<$ $\infty$. So, for each $F \in \mathcal{F}$, we can rewrite (1.1) as

$$
\xi(x)=\frac{\int_{(\alpha, x)} h(y) d(F(y))^{r}}{(F(x-))^{r}} .
$$

If we denote by $\mathcal{M}$ the set of these functions $\xi$, the expression (2.3) enables us to construct a map $\Psi: \mathcal{F} \rightarrow \mathcal{M}$, defined by $\Psi(F)=\xi$, and the objectives marked in the Introduction of this paper, may be rewritten as:

Q1: Is $\Psi$ a one-to-one map?

Q2: What is the explicit expression of $F=\Psi^{-1}(\xi)$ ?

Q3: What are the necessary and sufficient conditions in order for any real function to be in $\operatorname{Im}(\Psi)$ ?

Q4: Has $\Psi$ any type of continuity?

Analogously, if we consider the former questions for $\bar{\xi}$ given in (1.2), we obtain a parallel development for the order mean function

$$
\bar{\xi}(x)=-\frac{\int_{(x, \beta)} h(y) d(1-F(y))^{n-r}}{(1-F(x))^{n-r}} .
$$

To solve these questions, the concept of RSPI given in Zoroa and Ruiz [16] is essential (see Gill and Johansen [8] for a general study of product integral). So, we see now a modified definition of RSPI of Zoroa and Ruiz [16], and some results which we will subsequently use.

Definition 2.1. Let $f$ and $g$ be two real functions defined on $(a, b)$, with $0 \leq$ $f(x) \leq k$ and $g$ increasing and left continuous. We define the RSPI of $f$ with respect to $g$ in $(a, b)$, by

$$
\Lambda_{(a, b)}(1+f(x) d g(x))=\exp \left(J_{a b}\right)
$$

where $J_{a b} \in \mathbb{R}$, verifying that for all $\varepsilon>0$ there exists a $\delta>0$ such that if $\pi$ is a partition of $(a, b), \pi=\left\{a=x_{0}<x_{1}<\ldots<x_{m}=b\right\}$, with $g$-norm $N_{g}(\pi)=\max \left\{g\left(x_{j+1}\right)-g\left(x_{j}+\right): j=0,1, \ldots, m-1\right\}<\delta$, then

$$
\left|J(f, g, \pi)-J_{a b}\right|<\varepsilon,
$$

where

$$
\begin{aligned}
J(f, g, \pi)= & \sum_{j=0}^{m-1} \log \left(1+f\left(x_{j}^{\prime}\right)\left(g\left(x_{j+1}\right)-g\left(x_{j}+\right)\right)\right) \\
& +\sum_{j=1}^{m-1} \log \left(1+f\left(x_{j}+\right)\left(g\left(x_{j}+\right)-g\left(x_{j}\right)\right)\right)
\end{aligned}
$$

and $x_{j}^{\prime} \in\left[x_{j}, x_{j+1}\right]$ for all $j=0,1, \ldots, m-1$.

Definition 2.2. Let $f$ and $g$ be two functions satisfying the conditions in Definition 2.1. For each $c \in(a, b)$, we define the RSPI of $f$ with respect to $g$ on $c$ by

$$
\Lambda_{\{c\}}(1+f(x) d g(x))=\frac{\Lambda_{(a, b)}(1+f(x) d g(x))}{\Lambda_{(a, c)}(1+f(x) d g(x)) \underset{(c, b)}{\Lambda_{1}}(1+f(x) d g(x))} .
$$


Property 2.1. Let $f$ and $g$ be two functions satisfying the conditions in Definition 2.1. If $c \in(a, b)$, then $\underset{\{c\}}{\Lambda}(1+f(x) d g(x))=1+f(c+)(g(c+)-g(c))$.

Definition 2.3. Let $g:[a, b] \longrightarrow \mathbb{R}$ be a real, increasing and left continuous function, and let $A=\left\{a_{i}: i \in I\right.$ countable $\}$ be the set of discontinuity of $g$. We define the discrete component of $g$ by $g_{d}(x)=\sum_{a<a_{i}<x}\left(g\left(a_{i}+\right)-g\left(a_{i}\right)\right)$, and the continuous component of $g$ by $g_{c}(x)=g(x)-g_{d}(x)$.

Property 2.2. Let $f$ and $g$ be two functions satisfying the conditions in Definition 2.1. If there exists the Riemann-Stieltjes integral of $f$ with respect to the continuous component of $g$ in $(a, b)$, then for all $\varepsilon>0$ there exists a partition $\pi=\left\{a=x_{0}<\right.$ $\left.x_{1}<\ldots<x_{m}=b\right\}$ such that

$$
\sum_{j=0}^{m-1}\left|\varepsilon_{j}\right|<\varepsilon
$$

where $\varepsilon_{j}=\log \underset{\left(x_{j}, x_{j+1}\right)}{\Lambda}(1+f(x) d g(x))-\log \left(1+f\left(x_{j}^{\prime}\right)\left(g\left(x_{j+1}\right)-g\left(x_{j}+\right)\right)\right)$ and $x_{j}^{\prime} \in\left[x_{j}, x_{j+1}\right]$ for all $j=0,1, \ldots, m-1$.

Property 2.3. Let $f$ and $g$ be two functions satisfying the conditions in Definition 2.1 and such that there exists the Riemann-Stieltjes integral of $f$ with respect to $g_{c}$ in $(a, b)$. Then,

$$
\begin{aligned}
\underset{(a, b)}{\Lambda_{i}}(1+f(x) d g(x)) & \\
& =\exp \left(\int_{a}^{b} f(x) d g_{c}(x)+\sum_{a_{i} \in A} \log \left(1+f\left(a_{i}+\right)\left(g\left(a_{i}+\right)-g\left(a_{i}\right)\right)\right)\right)
\end{aligned}
$$

where $A=\left\{a_{i}\right\}$ is the set of discontinuity of $g(x)$ in $(a, b)$.

\section{Properties of the order mean function}

In this section, we show some properties of the order mean function $\xi$ defined in (2.3), which we will use as necessary conditions to characterize the order mean family $\mathcal{M}$.

Property 3.1. Let $F \in \mathcal{F}$ and $\xi=\Psi(F)$. Then the domain of $\xi$ is $D=(\alpha, \infty)$, where $\alpha \in \mathbb{R}$ or $\alpha=-\infty$.

Property 3.2. Let $F \in \mathcal{F}$ and $\xi=\Psi(F)$. If $h$ is strictly increasing (decreasing), then $\xi(x+)<h(x)(\xi(x+)>h(x))$ for all $x \in D$. Moreover, if $\alpha \in \mathbb{R}$, then $h(\alpha) \leq \xi(x+)(h(\alpha) \geq \xi(x+))$ for all $x \in D$.

Property 3.3. Let $F \in \mathcal{F}$ and $\xi=\Psi(F)$. Then $\xi$ is a left continuous function.

The proofs of Properties 3.1, 3.2 and 3.3 are obvious from the definition of order mean function given in (2.3).

Lemma 3.4. Let $F \in \mathcal{F}$ and $\xi=\Psi(F)$. If $x, y \in D$ with $x \leq y$, then

$$
\frac{h(x)-\xi(y)}{h(x)-\xi(x+)}-\frac{(F(x))^{r}}{(F(y-))^{r}}=\frac{(h(x)-h(\nu))\left((F(y-))^{r}-(F(x))^{r}\right)}{(h(x)-\xi(x+))(F(y-))^{r}}
$$


for some $\nu \in[x, y]$. Moreover,

$$
\frac{h(x)-\xi(y)}{h(x)-\xi(x+)} \leq\left(\frac{F(x)}{F(y-)}\right)^{r}
$$

where the equality holds if $F(x)=F(y-)$ or $x=y$.

Likewise, if $x<y$, then

$$
\frac{h(y)-\xi(x+)}{h(y)-\xi(y)} \leq\left(\frac{F(y-)}{F(x)}\right)^{r} .
$$

Proof. Let us suppose $h$ is strictly increasing (the case $h$ strictly decreasing is similar). If $x<y$, from (2.3) we obtain

$$
\xi(y)(F(y-))^{r}-\xi(x+)(F(x))^{r}=\int_{(x, y)} h(t) d(F(t))^{r},
$$

or equivalently,

$$
\frac{h(x)-\xi(y)}{h(x)-\xi(x+)}-\frac{(F(x))^{r}}{(F(y-))^{r}}=\frac{h(x)\left((F(y-))^{r}-(F(x))^{r}\right)-I}{(h(x)-\xi(x+))(F(y-))^{r}},
$$

where $I=\int_{(x, y)} h(t) d(F(t))^{r}$.

From the continuity of $h$ it follows that there exists an $\nu \in(x, y)$ such that $I=h(\nu)\left((F(y-))^{r}-(F(x))^{r}\right)$, and consequently, from (3.8) we have (3.5).

Hence, it is easy to see that

$$
\frac{(h(x)-h(\nu))\left((F(y-))^{r}-(F(x))^{r}\right)}{(h(x)-\xi(x+))(F(y-))^{r}} \leq 0 ;
$$

therefore, we get (3.6).

Furthermore, using the strict monotony of $h$, we obtain $h(x)-h(\nu)<0$. So, the equality of (3.6) holds when $F(x)=F(y-)$.

If $x=y$, from (2.3) we have

$$
\begin{aligned}
\xi(x+)(F(x))^{r}-\xi(x)(F(x-))^{r} & =\int_{(\alpha, x]} h(t) d(F(t))^{r}-\int_{(\alpha, x)} h(t) d(F(t))^{r} \\
& =h(x)\left((F(x))^{r}-(F(x-))^{r}\right)
\end{aligned}
$$

or equivalently,

$$
\frac{h(x)-\xi(x+)}{h(x)-\xi(x)}=\left(\frac{F(x-)}{F(x)}\right)^{r},
$$

i.e., the equality holds in (3.5) and (3.6).

Finally, using an analogous development we obtain

$$
\frac{(F(y-))^{r}}{(F(x))^{r}}-\frac{h(y)-\xi(x+)}{h(y)-\xi(y)}=\frac{(h(y)-h(\nu))\left((F(y-))^{r}-(F(x))^{r}\right)}{(h(y)-\xi(y))(F(x))^{r}},
$$

and consequently, we get (3.7).

Property 3.5. Let $F \in \mathcal{F}$ and $\xi=\Psi(F)$. If $h$ is strictly increasing (decreasing), then $\xi$ is increasing (decreasing). 
Proof. Let us suppose $h$ is strictly increasing (the case $h$ strictly decreasing is similar). From Property 3.2, for two points $a<b \in D$, we have $\xi(a+)<h(a+)=$ $h(a)$, and using Lemma 3.4, we obtain that

$$
\frac{h(a)-\xi(b)}{h(a)-\xi(a+)}=\frac{(F(a))^{r}}{(F(b-))^{r}} \quad \text { if } F(a)=F(b-),
$$

or

$$
\frac{h(a)-\xi(b)}{h(a)-\xi(a+)}<\frac{(F(a))^{r}}{(F(b-))^{r}}<1 \quad \text { if } F(a)<F(b-) ;
$$

so, if $F(a)=F(b-)$, then $\xi(a+)=\xi(b)$, or if $F(a)<F(b-)$, then $\xi(a+)<\xi(b)$.

\section{INVERSION FORMULA}

Let us now examine some previous lemmas, which will allow us to check the existence of the RSPI and to obtain the explicit expression of the distribution based on the order mean function.

Lemma 4.1. Let $F \in \mathcal{F}$ and $\xi=\Psi(F)$. For each $a \in D$, there exists a point $L_{a}=\inf \{|h(x)-\xi(x)|: x \geq a\}>0$.

Proof. Let us suppose $h$ is strictly increasing (the case $h$ strictly decreasing is similar). From (3.7), for $x>a$, we have

$$
(h(x)-\xi(a+))(F(a))^{r} \leq(h(x)-\xi(a+)) \frac{(F(a))^{r}}{(F(x-))^{r}} \leq h(x)-\xi(x) .
$$

Therefore, $h(x)-\xi(x)>(h(a)-\xi(a+))(F(a))^{r}>0$, and using Property 3.2,

$$
L_{a}=\inf \left\{(h(a)-\xi(a+))(F(a))^{r}, h(a)-\xi(a)\right\}>0 .
$$

Lemma 4.2. Let $F \in \mathcal{F}$ and $\xi=\Psi(F)$. For each $a \in D$, there exists an $\eta_{a}>0$ such that for all $b, c \in D$ with $a \leq b<c$ and $0<|h(b)-h(c)|<\eta_{a}$, the inequality $\eta_{a}<|h(b)-\xi(c)|$ is verified.

Proof. Let us suppose $h$ is strictly increasing (the case $h$ strictly decreasing is similar). If we consider $L_{a}$ given in Lemma 4.1 , we get $\eta_{a}>0$ such that $\eta_{a}<L_{a} / 2$, so we have

$$
h(b)-\xi(c)=-(h(c)-h(b))+h(c)-\xi(c) \geq-\eta_{a}+L_{a}>\frac{L_{a}}{2}>\eta_{a} .
$$

Theorem 4.3. Let $F \in \mathcal{F}$ and $\xi=\Psi(F)$. For all $(a, b) \subset D$ there exists the RSPI of $\frac{1}{h(x)-\xi(x)}$ with respect to $\xi(x)$ in $(a, b)$. Moreover,

$$
\left(\frac{F(b-)}{F(a)}\right)^{r}=\underset{(a, b)}{\Lambda}\left(1+\frac{d \xi(x)}{h(x)-\xi(x)}\right) .
$$

Proof. Let us suppose $h$ is strictly increasing (the case $h$ strictly decreasing is similar). From Property 3.2 and Lemma 4.2, we have that $f(x)=\frac{1}{h(x)-\xi(x)}$ is a nonnegative and bounded function in $(a, b)$. Therefore, $f$ is Riemann-Stieltjes integrable with respect to any continuous and monotonic function in $(a, b)$; in particular, with respect to the continuous component of $\xi$. Furthermore, from Properties 3.3 and $3.5, \xi$ is increasing and left continuous in $(a, b)$, so using Property 2.3 , we get the 
existence of the RSPI, i.e., there exists $J_{a b}$ such that for all $\varepsilon>0$ there exists a $\delta>0$ such that if $\pi=\left\{a=x_{0}<x_{1}<\ldots<x_{m}=b\right\}$ and $N_{\xi}(\pi)<\delta$, then

$$
\left|J\left(\frac{1}{h-\xi}, \xi, \pi\right)-J_{a b}\right|<\varepsilon,
$$

where

$$
J\left(\frac{1}{h-\xi}, \xi, \pi\right)=\sum_{j=0}^{m-1} \log \left(\frac{h\left(x_{j+1}\right)-\xi\left(x_{j}+\right)}{h\left(x_{j+1}\right)-\xi\left(x_{j+1}\right)}\right)+\sum_{j=1}^{m-1} \log \left(\frac{h\left(x_{j}\right)-\xi\left(x_{j}\right)}{h\left(x_{j}\right)-\xi\left(x_{j}+\right)}\right)
$$

and $x_{j}^{\prime}=x_{j+1}$ for all $j=0,1, \ldots, m-1$.

On the other hand, from (3.10) for $x=x_{j}$ and $y=x_{j+1}$, we have

$$
\begin{aligned}
0 & \leq \frac{\left(F\left(x_{j+1}-\right)\right)^{r}}{\left(F\left(x_{j}\right)\right)^{r}}-\frac{h\left(x_{j+1}\right)-\xi\left(x_{j}+\right)}{h\left(x_{j+1}\right)-\xi\left(x_{j+1}\right)} \\
& \leq \frac{\left(h\left(x_{j+1}\right)-h\left(x_{j}\right)\right)\left(\left(F\left(x_{j+1}-\right)\right)^{r}-\left(F\left(x_{j}\right)\right)^{r}\right)}{\left(h\left(x_{j+1}\right)-\xi\left(x_{j+1}\right)\right)\left(F\left(x_{j}\right)\right)^{r}}
\end{aligned}
$$

and taking into account that if $1 \leq x \leq y$, then $0 \leq \log y-\log x \leq y-x$, we obtain

$$
\begin{aligned}
0 & \leq \log \left(\frac{F\left(x_{j+1}-\right)}{F\left(x_{j}\right)}\right)^{r}-\log \left(\frac{h\left(x_{j+1}\right)-\xi\left(x_{j}+\right)}{h\left(x_{j+1}\right)-\xi\left(x_{j+1}\right)}\right) \\
& \leq \frac{\left(h\left(x_{j+1}\right)-h\left(x_{j}\right)\right)\left(\left(F\left(x_{j+1}-\right)\right)^{r}-\left(F\left(x_{j}\right)\right)^{r}\right)}{\left(h\left(x_{j+1}\right)-\xi\left(x_{j+1}\right)\right)\left(F\left(x_{j}\right)\right)^{r}} .
\end{aligned}
$$

Likewise, from (3.9) for $x=x_{j}$ we have

$$
\log \left(\frac{F\left(x_{j}\right)}{F\left(x_{j}-\right)}\right)^{r}-\log \left(\frac{h\left(x_{j}\right)-\xi\left(x_{j}\right)}{h\left(x_{j}\right)-\xi\left(x_{j}+\right)}\right)=0 .
$$

If we add from $j=0, \ldots, m-1$ in (4.13), from $j=1, \ldots, m-1$ in (4.14) and we take away (4.12), we obtain

$$
\begin{aligned}
0 & \leq \sum_{j=0}^{m-1} \log \left(\frac{F\left(x_{j+1}-\right)}{F\left(x_{j}\right)}\right)^{r}+\sum_{j=1}^{m-1} \log \left(\frac{F\left(x_{j}\right)}{F\left(x_{j}-\right)}\right)^{r}-J\left(\frac{1}{h-\xi}, \xi, \pi\right) \\
& \leq \sum_{j=0}^{m-1} \frac{\left(h\left(x_{j+1}\right)-h\left(x_{j}\right)\right)\left(\left(F\left(x_{j+1}-\right)\right)^{r}-\left(F\left(x_{j}\right)\right)^{r}\right)}{\left(h\left(x_{j+1}\right)-\xi\left(x_{j+1}\right)\right)\left(F\left(x_{j}\right)\right)^{r}},
\end{aligned}
$$

and using Lemma 4.1, we get the following bound:

$$
\log \left(\frac{F(b-)}{F(a)}\right)^{r}-J\left(\frac{1}{h-\xi}, \xi, \pi\right) \leq \frac{k_{a}\left((F(b-))^{r}-(F(a))^{r}\right)}{L_{a}(F(a))^{r}} \leq \frac{k_{a}}{L_{a}(F(a))^{r}},
$$

where $k_{a}=\sup \left\{h\left(x_{j+1}\right)-h\left(x_{j}\right): j=0,1, \ldots, m-1\right\}$.

So, from (4.11) and (4.15) we obtain that

$$
\left|\log \left(\frac{F(b-)}{F(a)}\right)^{r}-J_{a b}\right|<\varepsilon+\frac{k_{a}}{L_{a}(F(a))^{r}},
$$

and taking into account the continuity of $h$, we can take $\pi$ such that $k_{a}<$ $\varepsilon L_{a}(F(a))^{r}$. 
From Theorem 4.3 as $b \rightarrow \infty$, we obtain the distribution function for all points $x \in D$, which solves question (Q2) and consequently (Q1).

Corollary 4.4. Let $F \in \mathcal{F}$ and $\xi=\Psi(F)$. Then

$$
F(x)=\left(\underset{(x, \infty)}{\Lambda}\left(1+\frac{d \xi(y)}{h(y)-\xi(y)}\right)\right)^{-\frac{1}{r}}
$$

for all $x \in D$, adding $F(x)=0$ when $x<\alpha$.

Remark 4.1. Using Property 2.3, the distribution function given in (4.16) may be rewritten as

$$
F(x)=\exp \left(-\frac{1}{r} \int_{x}^{\infty} \frac{d \xi_{c}(y)}{h(y)-\xi(y)}\right)\left(\prod_{a_{i}>x} \frac{\xi\left(a_{i}+\right)-h\left(a_{i}\right)}{\xi\left(a_{i}\right)-h\left(a_{i}\right)}\right)^{\frac{1}{r}}
$$

where $\left\{a_{j}\right\}$ are the discontinuity points of $\xi$.

In particular, if $\xi$ is continuous, then (4.17) can be rewritten as

$$
F(x)=\exp \left(-\frac{1}{r} \int_{x}^{\infty} \frac{d \xi(y)}{h(y)-\xi(y)}\right)
$$

and this inversion formula includes the particular results of characterization given in Ferguson [5], Khan and Abu-Salih [9], Nagaraja [11] and Ouyang [14], as well as the derivable case given in Khan and Abu-Salih [9]; further it coincides with Theorem 3.2 of Franco and Ruiz [6].

In the case when $\xi$ is discrete, from (4.17) we get $F(x)=\left(\prod_{a_{i}>x} \frac{\xi\left(a_{i}+\right)-h\left(a_{i}\right)}{\xi\left(a_{i}\right)-h\left(a_{i}\right)}\right)^{\frac{1}{r}}$, which is the inversion formula for discrete distributions. Hence, we obtain the particular result of Nagaraja [12] for $n=2$; moreover this coincides with Theorem 6 of Franco and Ruiz [7].

On the other hand, if $h(x)=x$ and $r=1$, from (4.17), the inversion formula given in Zoroa and Ruiz [16] may be obtained.

Let us now see an immediate property from the above theorems, which will be basic in the following section.

Property 4.5. Let $F \in \mathcal{F}$ and $\xi=\Psi(F)$. Then,

(1) $\underset{(a, \infty)}{\Lambda}\left(1+\frac{d \xi(x)}{h(x)-\xi(x)}\right)$ converges for all $a \in D$.

(2) $\underset{(\alpha, \infty)}{\Lambda}\left(1+\frac{d \xi(x)}{h(x)-\xi(x)}\right)$ diverges.

(3) $\lim _{x \rightarrow \alpha} \xi(x+)\left(\underset{(x, \infty)}{\Lambda}\left(1+\frac{d \xi(y)}{h(y)-\xi(y)}\right)\right)^{-1}=0$.

Analogously, the following corollary shows the inversion formula based on $\bar{\xi}$.

Corollary 4.6. Let $\bar{\xi}$ be the order mean function associated to $F$ defined by (2.4). Then, $F(x)=1-\left(\underset{(-\infty, x]}{\Lambda}\left(1+\frac{d \bar{\xi}(y)}{\bar{\xi}(y)-h(y)}\right)\right)^{-\frac{1}{n-r}}$ for all $x \in \bar{D}$, adding $F(x)=1$ when $x>\beta$. 
Remark 4.2. Using Property 2.3, Corollary 4.6 may be rewritten as

$$
F(x)=1-\exp \left(-\frac{1}{n-r} \int_{-\infty}^{x} \frac{d \bar{\xi}_{c}(y)}{\bar{\xi}_{c}(y)-h(y)}\right)\left(\prod_{a_{i} \leq x} \frac{\bar{\xi}\left(a_{i}-\right)-h\left(a_{i}\right)}{\bar{\xi}\left(a_{i}\right)-h\left(a_{i}\right)}\right)^{\frac{1}{n-r}}
$$

where $\left\{a_{j}\right\}$ are the discontinuity points of $\bar{\xi}$.

In particular, if $\bar{\xi}$ is continuous, then $F(x)=1-\exp \left(-\frac{1}{n-r} \int_{-\infty}^{x} \frac{d \bar{\xi}(y)}{\bar{\xi}(y)-h(y)}\right)$, which includes some particular characterizations given by Ferguson [5], Khan and Abu-Salih [9], Nagaraja [11] and Ouyang [13, 14], as well as the derivable case given by Khan and Abu-Salih [9]. Moreover this coincides with Theorem 3.3 of Franco and Ruiz [6].

In the case when $\bar{\xi}$ is discrete, from (4.18) we get

$$
F(x)=1-\left(\prod_{a_{i} \leq x} \frac{\bar{\xi}\left(a_{i}-\right)-h\left(a_{i}\right)}{\bar{\xi}\left(a_{i}\right)-h\left(a_{i}\right)}\right)^{\frac{1}{n-r}} .
$$

Hence, we deduce Theorem 2 of Nagaraja [12] when $h(x)=x, n=2$ and $\bar{\xi}$ is linear. Further this formula coincides with the expression (20) of Franco and Ruiz [7].

\section{NECESSARY AND SUfFiciEnT CONDITIONS}

To solve (Q3), we give in the following theorem necessary and sufficient conditions so that any real function belongs to $\mathcal{M}$.

Theorem 5.1. Let $\xi$ be a real function. Then $\xi$ is an order mean function if and only if $\xi$ satisfies the following conditions:

(1) The domain of $\xi$ is $D=(\alpha, \infty)$, where $\alpha \in \mathbb{R}$ or $\alpha=-\infty$.

(2) If $h$ is strictly increasing (decreasing), then $\xi(x+)<h(x)(\xi(x+)>h(x))$ for all $x \in D$. Moreover, if $\alpha \in \mathbb{R}$, then $h(\alpha) \leq \xi(x+)(h(\alpha) \geq \xi(x+))$ for all $x \in D$.

(3) If $h$ is strictly increasing (decreasing), then $\xi$ is increasing (decreasing).

(4) $\xi$ is left continuous in $D$.

(5) $\underset{(a, \infty)}{\Lambda}\left(1+\frac{d \xi(x)}{h(x)-\xi(x)}\right)$ converges for all $a \in D$.

(6) $\underset{(\alpha, \infty)}{\Lambda}\left(1+\frac{d \xi(x)}{h(x)-\xi(x)}\right)$ diverges.

(7) $\lim _{x \rightarrow \alpha} \xi(x+)\left(\underset{(x, \infty)}{\Lambda}\left(1+\frac{d \xi(y)}{h(y)-\xi(y)}\right)\right)^{-1}=0$.

Proof. We have shown that all these conditions are necessary. To prove that they are sufficient, let us consider a real function $\xi$ whose domain of definition we denote by $D$, and which satisfies conditions of the statement. Let us define the function

$$
G(x)=\left(\underset{(x, \infty)}{\Lambda}\left(1+\frac{d \xi(y)}{h(y)-\xi(y)}\right)\right)^{-\frac{1}{r}}
$$

for all $x \in D$, adding $G(x)=0$ when $x<\alpha$.

Let us first see that $G$ is a distribution function. From (5) the definition of $G$ makes sense for all $x \in D$, and from (6) we have that $\lim _{x \rightarrow \alpha} G(x)=0$. It is easy to 
prove that

$$
\Lambda_{(x, \infty)}\left(1+\frac{d \xi(y)}{h(y)-\xi(y)}\right)=\frac{\Lambda_{(a, \infty)}\left(1+\frac{d \xi(y)}{h(y)-\xi(y)}\right)}{\Lambda_{(a, x)}\left(1+\frac{d \xi(y)}{h(y)-\xi(y)}\right) \Lambda_{\{x\}}\left(1+\frac{d \xi(y)}{h(y)-\xi(y)}\right)} .
$$

From the above equality, as $x \rightarrow \infty$, we get $\lim _{x \rightarrow \infty} \underset{(x, \infty)}{\Lambda}\left(1+\frac{d \xi(y)}{h(y)-\xi(y)}\right)=1$, i.e., $\lim _{x \rightarrow \infty} G(x)=1$.

On the other hand, from (2) and (3) we obtain that $G$ is increasing. Likewise, from (4) and taking into account that

$$
G(x)=G\left(x_{1}\right)\left(\underset{\left(x_{1}, x\right)}{\Lambda}\left(1+\frac{d \xi(y)}{h(y)-\xi(y)}\right) \frac{h(x)-\xi(x)}{h(x)-\xi(x+)}\right)^{\frac{1}{r}},
$$

we obtain that $\lim _{x \rightarrow x_{1}^{+}} G(x)=G\left(x_{1}\right)$, i.e., $G$ is right continuous.

Consequently, $G$ is a distribution function, and therefore $\int_{(a, b)} h(y) d(G(y))^{r}$ exists for all $(a, b) \subset D$.

On the other hand, we prove the following identity:

$$
\xi(b)(G(b-))^{r}-\xi(a+)(G(a))^{r}=\int_{(a, b)} h(y) d(G(y))^{r} .
$$

To establish (5.19) we suppose $h$ strictly increasing (the case $h$ strictly decreasing is similar), and let $\pi$ be a partition of $(a, b)$. Then

$$
\begin{aligned}
& \xi(b)(G(b-))^{r}-\xi(a+)(G(a))^{r} \\
& =\sum_{j=0}^{m-1}\left(\xi\left(x_{j+1}\right)\left(\left(G\left(x_{j+1}-\right)\right)^{r}-\left(G\left(x_{j}\right)\right)^{r}\right)+\left(G\left(x_{j}\right)\right)^{r}\left(\xi\left(x_{j+1}\right)-\xi\left(x_{j}+\right)\right)\right) \\
& +\sum_{j=1}^{m-1}\left(\xi\left(x_{j}+\right)\left(G\left(x_{j}\right)\right)^{r}-\xi\left(x_{j}\right)\left(G\left(x_{j}-\right)\right)^{r}\right) .
\end{aligned}
$$

Thus,

$$
\int_{(a, b)} h(y) d(G(y))^{r}-\left(\xi(b)(G(b-))^{r}-\xi(a+)(G(a))^{r}\right)=P_{1}+P_{2},
$$

where

$$
\begin{aligned}
P_{1}= & \int_{(a, b)} h(y) d(G(y))^{r}-\sum_{j=0}^{m-1} h\left(x_{j+1}\right)\left(\left(G\left(x_{j+1}-\right)\right)^{r}-\left(G\left(x_{j}\right)\right)^{r}\right) \\
& -\sum_{j=1}^{m-1} h\left(x_{j}\right)\left(\left(G\left(x_{j}\right)\right)^{r}-\left(G\left(x_{j}-\right)\right)^{r}\right)
\end{aligned}
$$


and

$$
\begin{aligned}
P_{2}= & \sum_{j=0}^{m-1}\left(h\left(x_{j+1}\right)-\xi\left(x_{j+1}\right)\right)\left(\left(G\left(x_{j+1}-\right)\right)^{r}-\left(G\left(x_{j}\right)\right)^{r}\right) \\
& -\sum_{j=0}^{m-1}\left(G\left(x_{j}\right)\right)^{r}\left(\xi\left(x_{j+1}\right)-\xi\left(x_{j}+\right)\right) \\
& +\sum_{j=1}^{m-1}\left(\left(h\left(x_{j}\right)-\xi\left(x_{j}+\right)\right)\left(G\left(x_{j}\right)\right)^{r}-\left(h\left(x_{j}\right)-\xi\left(x_{j}\right)\right)\left(G\left(x_{j}-\right)\right)^{r}\right) .
\end{aligned}
$$

Let us first see that $P_{2}$ is bounded. From Property 2.1 and using the definition of $G$, we have

$$
\frac{h\left(x_{j}\right)-\xi\left(x_{j}\right)}{h\left(x_{j}\right)-\xi\left(x_{j}+\right)}=\frac{\underset{\left[x_{j}, \infty\right)}{\Lambda}\left(1+\frac{d \xi(y)}{h(y)-\xi(y)}\right)}{\underset{\left(x_{j}, \infty\right)}{\Lambda}\left(1+\frac{d \xi(y)}{h(y)-\xi(y)}\right)}=\frac{\left(G\left(x_{j}\right)\right)^{r}}{\left(G\left(x_{j}-\right)\right)^{r}} .
$$

So, the last sum in the expression for $P_{2}$ is null, and we can write it as

$$
\begin{aligned}
P_{2}= & \sum_{j=0}^{m-1}\left(h\left(x_{j+1}\right)-\xi\left(x_{j+1}\right)\right)\left(G\left(x_{j}\right)\right)^{r}\left(\frac{\left(G\left(x_{j+1}-\right)\right)^{r}}{\left(G\left(x_{j}\right)\right)^{r}}-1\right) \\
& -\sum_{j=0}^{m-1}\left(G\left(x_{j}\right)\right)^{r}\left(\xi\left(x_{j+1}\right)-\xi\left(x_{j}+\right)\right)
\end{aligned}
$$

which can be transformed to

$$
\begin{aligned}
P_{2}= & \sum_{j=0}^{m-1}\left(G\left(x_{j}\right)\right)^{r}\left(h\left(x_{j+1}\right)-\xi\left(x_{j+1}\right)\right)\left(\underset{\left(x_{j}, x_{j+1}\right)}{\Lambda}\left(1+\frac{d \xi(x)}{h(x)-\xi(x)}\right)-1\right) \\
& -\sum_{j=0}^{m-1}\left(G\left(x_{j}\right)\right)^{r}\left(\xi\left(x_{j+1}\right)-\xi\left(x_{j}+\right)\right) .
\end{aligned}
$$

Now, from Property 2.2, for each $\varepsilon>0$ there exists a partition $\pi$ such that $\sum_{j=0}^{m-1}\left|\varepsilon_{j}\right|<\varepsilon$, where $\exp \varepsilon_{j}=\left(1+\frac{\xi\left(x_{j+1}\right)-\xi\left(x_{j}+\right)}{h\left(x_{j+1}\right)-\xi\left(x_{j+1}\right)}\right)^{-1} \underset{\left(x_{j}, x_{j+1}\right)}{\Lambda}\left(1+\frac{d \xi(x)}{h(x)-\xi(x)}\right)$, and $x_{j}^{\prime}=x_{j+1}$ for all $j=0,1, \ldots, m-1$. So,

$$
\begin{aligned}
& \underset{\left(x_{j}, x_{j+1}\right)}{\Lambda}\left(1+\frac{d \xi(x)}{h(x)-\xi(x)}\right) \\
& \quad=\left(1+\frac{\xi\left(x_{j+1}\right)-\xi\left(x_{j}+\right)}{h\left(x_{j+1}\right)-\xi\left(x_{j+1}\right)}\right)\left(1+\left(\exp \varepsilon_{j}-1\right)\right) \\
& \quad=1+\frac{\xi\left(x_{j+1}\right)-\xi\left(x_{j}+\right)}{h\left(x_{j+1}\right)-\xi\left(x_{j+1}\right)}+\left(\exp \varepsilon_{j}-1\right)\left(\frac{h\left(x_{j+1}\right)-\xi\left(x_{j}+\right)}{h\left(x_{j+1}\right)-\xi\left(x_{j+1}\right)}\right)
\end{aligned}
$$

which transforms (5.20) to

$$
\left|P_{2}\right| \leq \sum_{j=0}^{m-1}\left(G\left(x_{j}\right)\right)^{r}\left(h\left(x_{j+1}\right)-\xi\left(x_{j}+\right)\right)\left|\exp \varepsilon_{j}-1\right| .
$$


Taking into account that for each $j,\left|\exp \varepsilon_{j}-1\right|=\left|\varepsilon_{j} \exp \left(\theta \varepsilon_{j}\right)\right| \leq\left|\varepsilon_{j}\right| \exp \left|\varepsilon_{j}\right|$ for any $0<\theta<1$, we get for $\varepsilon<1$, the bound

$$
\left|P_{2}\right| \leq(h(b)-\xi(a+)) e \sum_{j=0}^{m-1}\left|\varepsilon_{j}\right|<(h(b)-\xi(a+)) e \varepsilon .
$$

On the other hand, from the definition of the Lebesgue-Stieltjes integral, for each $\varepsilon>0$ there exists a partition $\pi$ such that $\left|P_{1}\right|<\varepsilon$.

Consequently, (5.19) is verified. Furthermore, from (7), if we take the limit as $a \rightarrow \alpha$ in (5.19), we have $\xi(b)(G(b-))^{r}=\int_{(\alpha, b)} h(y) d(G(y))^{r}$, which proves that $G \in \mathcal{F}$ and $\xi$ is its associated order mean function.

\section{Stability theorems}

In this section we see a continuity type of the map $\Psi$ defined in Section 2 , so we solve the last objective (Q4) of this paper through the following theorems.

Theorem 6.1. Let $\xi=\Psi(F)$ and $\xi_{n}=\Psi\left(F_{n}\right)$ for all $n \in \mathbb{N}$, be order mean functions with domains $D$ and $D_{n}$, respectively. If $F_{n}$ weakly converges to $F\left(F_{n} \stackrel{w}{\rightarrow}\right.$ $F)$, then $\xi_{n} \stackrel{w}{\rightarrow} \xi$.

Proof. In fact, if $x \in D$ is a continuity point of $\xi$, then $x$ is a continuity point of $F$. As $F_{n} \rightarrow F$ in $x$, there exists an $n_{0}$ such that if $n>n_{0}$, then $x \in D_{n}$.

Furthermore, $\left(F_{n}\right)^{r} \stackrel{w}{\rightarrow} F^{r}$ and $\left(F_{n}\right)^{r}$ are increasing and right continuous functions, so using Helly's second theorem, we have that $\lim _{n} \int_{(-\infty, x)} h(y) d\left(F_{n}(y)\right)^{r}$ $=\int_{(-\infty, x)} h(y) d(F(y))^{r}$. Therefore,

$$
\lim _{n} \xi_{n}(x)=\lim _{n} \frac{\int_{(-\infty, x)} h(y) d\left(F_{n}(y)\right)^{r}}{\left(F_{n}(x-)\right)^{r}}=\frac{\int_{(-\infty, x)} h(y) d(F(y))^{r}}{(F(x-))^{r}}=\xi(x) .
$$

Theorem 6.2. Let $\xi=\Psi(F)$ and $\xi_{n}=\Psi\left(F_{n}\right)$ for all $n \in \mathbb{N}$ be order mean functions with domains $D$ and $D_{n}$, respectively. If $\lim _{x \rightarrow \alpha} \liminf _{n} F_{n}(x)=0, \lim _{x \rightarrow \beta} \limsup _{n} F_{n}(x)$ $=1$ and $\xi_{n} \stackrel{w}{\rightarrow} \xi$, then $F_{n} \stackrel{w}{\rightarrow} F$.

Proof. Let us suppose that $F_{n} \stackrel{w}{\rightarrow} F$, so there exists a continuity point $x_{0} \in D$ of $F$ such that $F_{n}\left(x_{0}\right) \nrightarrow F\left(x_{0}\right)$, i.e., there exists a subsequence $\left(F_{n_{k}}\right)$ such that $\lim _{k} F_{n_{k}}\left(x_{0}\right) \neq F\left(x_{0}\right)$. Likewise, $F_{n_{k}}$ are increasing and uniformly bound, and using Helly's first theorem, $\left(F_{n_{k}}\right)$ has a subsequence $\left(F_{n_{k_{j}}}\right)$ weakly convergent to an increasing function $G$ with $\lim _{j} F_{n_{k_{j}}}\left(x_{0}\right)=G\left(x_{0}\right) \neq F\left(x_{0}\right)$, and $G$ is right continuous and $0 \leq G(x) \leq 1$.

Moreover, $0 \leq \lim _{x \rightarrow \alpha} G(x) \leq \lim _{x \rightarrow \alpha} \limsup _{n_{k}} F_{n_{k}}(x)=0$, and analogously, $\lim _{x \rightarrow \alpha} G(x)=$ 1. Therefore, $G(x)$ is a distribution function with support $(\alpha, \beta)$. If we denote its associated order mean function by $\xi_{G}$, from Theorem 6.1 we have that $\xi_{n_{k_{j}}} \stackrel{w}{\rightarrow} \xi_{G}$. Nevertheless, $\xi_{n} \stackrel{w}{\rightarrow} \xi$, the continuity points of $\xi$ are dense in $(\alpha, \beta)$, and $\xi$ and $\xi_{G}$ are left continuous, therefore $\xi=\xi_{G}$. And using Corollary 4.4, we obtain that $F=G$, which stands in contradiction to $G\left(x_{0}\right) \neq F\left(x_{0}\right)$. 


\section{EXAMPLES}

As we saw in Section 4, one can characterize several particular distributions in the discrete and continuous cases from results of this work, so we have obtained a unified approach. Moreover, we can get new characterizations for mixture distributions, as in the two following examples.

Example 7.1. Let $h(x)=x$ and let $\xi$ be given by

$$
\xi(x)=\frac{\lambda p\left(1-e^{-\theta x}(1+\theta x)\right)+(1-\lambda) \theta(1-p)\left(1-(1-p)^{[x]}(1+[x] p)\right)}{\theta p\left(1-\lambda e^{-\theta x}-(1-\lambda)(1-p)^{\langle x\rangle}\right)},
$$

where $\langle x\rangle=\inf \{m \in \mathbb{Z} \mid m \geq x\}$ and $[x]$ is the integer part of $x$. It is easy to prove that $\xi$ verifies Theorem 5.1, i.e., $\xi$ is an order mean function. Then using Corollary 4.4 for $r=1$, we have that $\xi$ characterizes the distribution $F$ given by the mixture $F(x)=\lambda F_{1}(x)+(1-\lambda) F_{2}(x)$, where $0 \leq \lambda \leq 1, F_{1}$ is the exponential distribution of parameter $\theta>0$ and $F_{2}$ is the geometric distribution of parameter $0<p<1$, i.e., $F_{1}(x)=1-e^{-\theta x}$ and $F_{2}(x)=1-(1-p)^{[x]+1}$ for all $x>0$.

In particular, if $\lambda=1$ we obtain the exponential distribution given in Ferguson [5] and Nagaraja [11], and if $\lambda=0$ we have the geometric distribution given in Nagaraja [12] for $n=2$ and in Franco and Ruiz [7] for $n>2$.

Example 7.2. Let $h(x)=x$ and let $\bar{\xi}$ be given by

$$
\bar{\xi}(x)=\frac{\lambda p(1+\theta x) e^{-\theta x}+(1-\lambda) \theta(1+[x] p)(1-p)^{[x]+1}}{\theta p\left(\lambda e^{-\theta x}+(1-\lambda)(1-p)^{[x]+1}\right)} .
$$

It is straightforward to check that $\bar{\xi}$ is an order mean function, and using Corollary 4.6 for $r=n-1$, we obtain that $\bar{\xi}$ characterizes the mixture of Example 7.1. In particular, if $\lambda=1$, then we obtain the characterization given by Khan and Abu-Salih [9] and Rogers [15], and if $\lambda=0$, then we obtain the characterization given by Nagaraja [12].

\section{ACKNOWLEDGEMENT}

The authors are thankful to the referee for the careful reading of this paper.

\section{REFERENCES}

[1] B.C. Arnold, Two characterizations of the geometric distribution, J. Appl. Probab., 17 (1980), 570-573. MR 82h:62024

[2] B.C. Arnold, N. Balakrishnan and H.N. Nagaraja, A first course in order statistics, Wiley, New York, 1992. MR 94a:62076

[3] B.C. Arnold, A. Becker, U. Gather and H. Zahedi, On the Markov property of order statistics, J. Statist. Plann. Inference, 9 (1984), 147-154. MR 85h:62011

[4] E. El-Neweihi and Z. Govindarajulu, Characterizations of geometric distribution and discrete IFR (DFR) distributions using order statistics, J. Statist. Plann. Inference, 3 (1979), 85-90. MR 81a:62012

[5] T.S. Ferguson, On characterizing distributions by properties of order statistics, Sankhyā Ser. A, 29 (1967), 265-278. MR 37:2391

[6] M. Franco and J.M. Ruiz, On characterization of continuous distributions with adjacent order statistics, Statistics, 26 (1995), 375-385. CMP 96:05

[7] M. Franco and J.M. Ruiz, Characterization of discrete populations through conditional expectations of order statistics, Statist. Papers (in press).

[8] R.D. Gill and S. Johansen, A survey of product integration with a view toward application in survival analysis, Ann. Statist., 4 (1990), 1501-1555. MR 92f:60125 
[9] A.H. Khan and M.S. Abu-Salih, Characterizations of probability distributions by conditional expectations of order statistics, Metron, 47 (1989), 171-181. CMP 91:10

[10] H.N. Nagaraja, On the non-markovian structure of discrete order statistics, J. Statist. Plann. Inference, 7 (1982), 29-33. MR 84a:62075

[11] H.N. Nagaraja, Some characterizations of continuous distributions based on regressions of adjacent order statistics and record values, Sankhyā Ser. A, 50 (1988a), 70-73. MR 91e:62035

[12] H.N. Nagaraja, Some characterizations of discrete distributions based on linear regressions of adjacent order statistics, J. Statist. Plann. Inference, 20 (1988b), 65-75. MR 90a:62046

[13] L.Y. Ouyang, Characterizations of the uniform distribution by conditional expectations, Internat. J. Inform. Management Sci., 4 (1993), 107-111. MR 94g:62020

[14] L.Y. Ouyang, Characterization through the conditional expectation of a function of one order statistic relative to an adjacent one, Sankhyā Ser. A, 57 (1995), 500-503. CMP 96:13

[15] G.S. Rogers, An alternative proof of the characterization of the density $A x^{B}$, Amer. Math. Monthly, 70 (1963), 857-858.

[16] P. Zoroa and J.M. Ruiz, El producto integral de Riemann-Stieltjes y su aplicación a las funciones de medias de las distribuciones truncadas, Rev. Real Acad. Cienc. Exact. Fís. Natur. Madrid, 79 (1985), 39-51. MR 87f:60024

Departamento de Estadística e I.O., Universidad de Murcia, 30100 Murcia, Spain

E-mail address: mfranco@fu.um.es

E-mail address: jmruizgo@fcu.um.es 